Research Article

\title{
A New Analytical Method for Spherical Thin Shells' Axisymmetric Vibrations
}

\author{
Mariame Nassit $\mathbb{D}^{\mathrm{D}},{ }^{1}$ Abderrahmane El Harif $\mathbb{D}^{2},{ }^{2}$ Hassan Berbia $\mathbb{D}^{1},{ }^{1}$ \\ and Mourad Taha Janan $\mathbb{1}^{3}$ \\ ${ }^{1}$ ENSIAS, Mohammed V University in Rabat, Rabat, Morocco \\ ${ }^{2}$ Faculty of Science, Mohammed V University in Rabat, Rabat, Morocco \\ ${ }^{3}$ ENSET, Mohammed $V$ University in Rabat, Rabat, Morocco \\ Correspondence should be addressed to Mariame Nassit; mariame_nassit@um5.ac.ma
}

Received 16 July 2020; Revised 19 October 2020; Accepted 2 November 2020; Published 30 November 2020

Academic Editor: Francisco J. Montáns

Copyright (c) 2020 Mariame Nassit et al. This is an open access article distributed under the Creative Commons Attribution License, which permits unrestricted use, distribution, and reproduction in any medium, provided the original work is properly cited.

In order to improve the spherical thin shells' vibrations analysis, we introduce a new analytical method. In this method, we take into consideration the terms of the inertial couples in the stress couples' differential equations of motion. These inertial couples are omitted in the theories provided by Naghdi-Kalnins and Kunieda. The results show that the current method can solve the axisymmetric vibrations' equations of elastic thin spherical shells. In this paper, we focus on verifying the current method, particularly for free vibrations with free edge and clamped edge boundary conditions. To check the validity and accuracy of the current analytical method, the natural frequencies determined by this method are compared with those available in the literature and those obtained by a finite element calculation.

\section{Introduction}

With the fast and continuous advancement of technology, it becomes necessary to have more efficient and effective innovative devices and engineering structures. Many publications have focused on modeling lightweight structures [1-3]. Thin spherical shells are used in civil engineering and a variety of engineering applications such as submarine hulls, aircraft, smart devices, and health monitoring systems [4-7]. It is well known that the spherical shells can be used in complex environmental conditions and can be exposed to a variety of dynamic excitations inducing excessive vibrations [8]. Undesirable vibrations cause structural failure, cracks in parts of machine elements, malfunction of electronic devices, and many other issues [9]. By measuring structure or system frequencies, designers can select speeds remote from resonance and can prevent many adverse effects of vibration [9]. Determining the frequency spectrum of a structure is an essential step of its design. In addition, developing an accurate and unified analytical solution for axisymmetric thin spherical shells with free edge or clamped edge boundary conditions remains an extremely challenging task. This solution is useful to provide reference data for future research in related fields. Hence, a survey of the literature depicts the existence of many studies on free vibrations analysis [10-14]. Besides, the research for curved shells, especially for cylindrical and spherical shells, have attracted much attention recently $[7,15-20]$.

Shell structures' vibrations analysis can be carried out by analytical or numerical methods and can be tackled by different theories and approximations. The linear theories for plates and shells are as follows:

(i) Love-Kirchhoff's theory that does not take account of transverse shear deformation that generalizes Euler-Bernoulli's theory for beams [21-23].

(ii) Mindlin-Reissner's theory for plates and Naghdi-Reissner's one for shells, taking into account the transverse shear deformation extending Timoshenko's theory for beams [24-30].

They all assume an elastic behavior. They are said to be of the first-order: the displacement fields vary linearly with 
variable $t$ (Figure 1 ) in the thickness, while the thickness does not vary.

However, higher-order theories have been developed like the theory HSDTs (Reddy) and many others [31].

Exact (Naghdi-Kalnins, Kunieda, current method) or approximate (Kunieda) analytical resolution methods are based on Legendre functions [32-34]. One cites among the numerical methods, finite differences, weighted residuals, variational methods (Rayleigh-Ritz), and finite element method [35-38].

Using the finite element method, El Harif studied the transverse shear effect in plates and shells of revolution [39]. Batoz et al. focused on structure modeling [40]. Juber investigated the analytical solution for the dynamic analysis of thin composite cylindrical and spherical shells. He presented the fundamental natural frequencies and mode shapes for simply supported composite cylindrical and spherical shells, by applying the third-order theory of Reddy [41]. Moreover, Sammoura et al. developed an analytical solution for curved piezoelectric micromachined ultrasonic transducers by applying Naghdi-Kalnins' theory [17].

The present paper concentrate on a new analytical method inspired by the Naghdi-Kalnins' theory [32] and the model presented in $[39,40]$. This method solves elastic thin spherical shells' axisymmetric vibrations' differential equations. For this purpose, we introduce the differential equations of motion in terms of stress, where we take into consideration the terms of the inertial couples in the stress couples' equations. These terms are omitted in the analytical methods proposed by Naghdi-Kalnins and Kunieda [32, 33]. Then, we introduce a new stress function different from Naghdi-Kalnins' one and derivate new equations depending on the displacement and the stress function. Moreover, by using the Legendre functions, we analytically solve these differential equations in the specific case of the hemispherical shell's harmonic axisymmetric vibrations, under the free and clamped edges boundary conditions. To check the validity and accuracy of the current method, we compare the current analytical results with Naghdi-Kalnins' results [32] and Kunieda's results [33]. It is worth to mention that Naghdi-Kalnins' theory is one of the most important references used in different recent studies [17, 42, 43]. After that, we compare the results with the finite element method (FEM) ones. The FEM numerical simulation results confirm the correctness and accuracy of the current analytical method.

The remainder of this paper is organized as follows: in Section 2, we introduce the analytical model formulation. In Section 3, we propose the general solution for harmonic axisymmetric vibration. After that, we present and discuss the results in Section 4. The conclusions of this study are described in Section 5.

\section{Analytical Model Formulation}

2.1. Basic Concept. Inspired by Naghdi-Kalnins' theory of axisymmetric vibrations of the elastic spherical shell, we introduce here new basic concepts that are used to analyze the axisymmetric vibrations response of the elastic spherical shells as illustrated in Figure 2. The main purpose

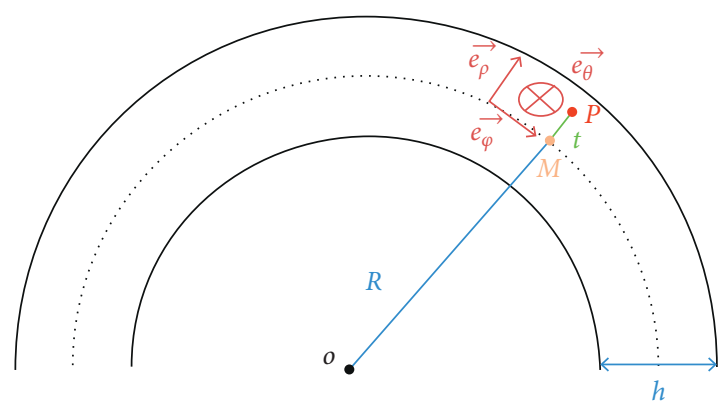

FIgURE 1: The thin spherical shell's thickness in the spherical coordinate system.

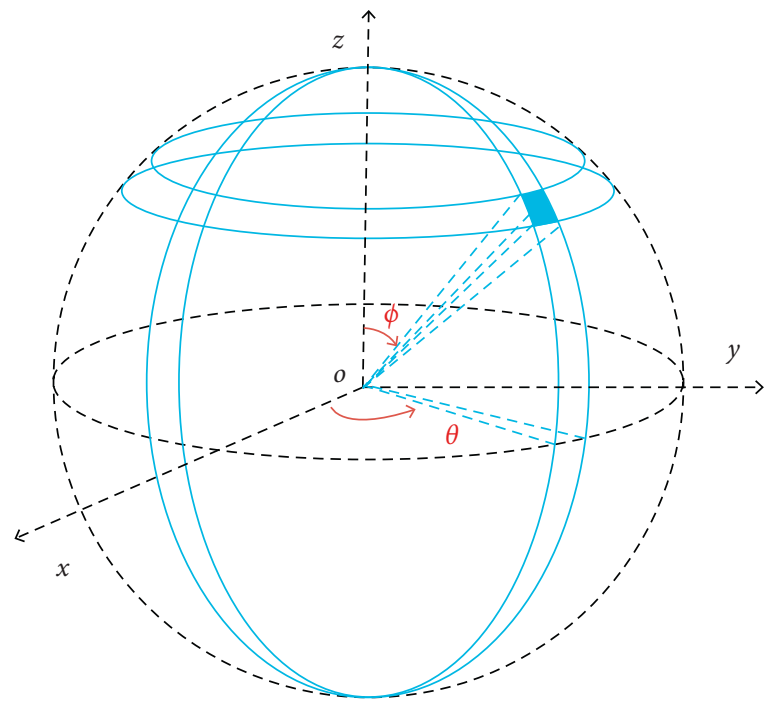

Figure 2: Middle surface element of the thin spherical shell.

of the approaches presented below is to derivate the expression of the differential equations by applying the motion equations with their stress couples equations. These stress couples equations take into account the terms of the inertial couples. These terms are omitted by Naghdi-Kalnins. In addition, we propose a new stress function that helps to derivate the new equations, different from Naghdi-Kalnins' equations. On the other side, as the thickness of the shells $h$ is small in comparison with the radius $R(h / R \ll 1)$, we use Love's first-order approximation for the dynamic analysis of the thin spherical shell. We assume that the transverse shear and normal strains are negligible. We recall here that the basic equations characterize the elastic deformation of the spherical shell in the weightlessness and the spherical coordinate system $(\rho, \theta, \varphi)$.

\subsection{Strain-Displacement Relations in Spherical Coordinates.} By applying Love-Kirchhoff's assumption, the strain can be expressed as a function of the displacement vector components and their derivatives in the spherical coordinate system, where $u_{\varphi}, u_{\theta}$, and $w$ are the displacement vector components in $\varphi-, \theta-$, and $\rho$ - directions, respectively. 
Furthermore, the total strain of a spherical shell can be expressed into two components: membrane strains $\varepsilon_{i j}^{0}$ and flexural strains $\varepsilon_{i j}^{1}$ as mentioned in [44]:

$$
\varepsilon_{i j}=\varepsilon_{i j}^{0}+t \varepsilon_{i j}^{1},
$$

where $i, j \in\{\varphi, \theta\}$.

As shown in Figure 1, $t$ is the radial position measured from the middle surface along the spherical shell thickness, where

$$
\begin{aligned}
& \frac{-h}{2} \leq t \leq \frac{h}{2} . \\
& \overrightarrow{O M}=R \overrightarrow{e_{\rho}}, \\
& \overrightarrow{O P}=R \overrightarrow{e_{\rho}}+t \overrightarrow{e_{\rho}},
\end{aligned}
$$

where $M$ is a point on the middle surface.

The membrane and the flexural strains are given by the following expressions [44]:

$\varepsilon_{\varphi \varphi}^{0}=\frac{1}{R}\left(u_{\varphi, \varphi}+w\right)$,

$\varepsilon_{\theta \theta}^{0}=\frac{1}{R}\left(\cot \varphi u_{\varphi}+\frac{1}{\sin \varphi} u_{\theta, \theta}+w\right)$,

$2 \varepsilon_{\theta \varphi}^{0}=\frac{1}{R}\left(u_{\theta, \varphi}-\cot \varphi u_{\varphi}+\frac{1}{\sin \varphi} u_{\varphi, \theta}\right)$,

$\varepsilon_{\varphi \varphi}^{1}=\frac{1}{R^{2}}\left(u_{\varphi, \varphi}-w_{, \varphi \varphi}\right)$,

$\varepsilon_{\theta \theta}^{1}=\frac{1}{R^{2}}\left(\cot \varphi u_{\varphi}+\frac{1}{\sin \varphi} u_{\theta, \theta}-\cot \varphi w_{, \varphi}-\frac{1}{\sin ^{2} \varphi} w_{, \theta \theta}\right)$,

$2 \varepsilon_{\theta \varphi}^{1}=\frac{1}{R^{2}}\left(\frac{1}{\sin \varphi} u_{\varphi, \theta}-\cot \varphi u_{\theta}+u_{\theta, \varphi}-\frac{2}{\sin \varphi^{2}} w_{, \varphi \theta}+2 \frac{\cot \varphi}{\sin \varphi} w_{, \theta}\right)$.

2.3. Stress Resultants and Stress Couples in Spherical Coordinate System. The tangential stresses of the spherical shells in spherical coordinates can be expressed as

$$
\begin{aligned}
& \sigma_{\varphi \varphi}=\frac{E}{1-\nu^{2}}\left[\varepsilon_{\varphi \varphi}+\nu \varepsilon_{\theta \theta}\right], \\
& \sigma_{\theta \theta}=\frac{E}{1-\nu^{2}}\left[\varepsilon_{\theta \theta}+\nu \varepsilon_{\varphi \varphi}\right], \\
& \sigma_{\varphi \theta}=\sigma_{\theta \varphi}=\frac{E}{1-\nu^{2}} \varepsilon_{\theta \varphi},
\end{aligned}
$$

where $E$ is Young's modulus, $v$ is Poisson's ratio.

As shown in Figure 3, it is convenient to introduce the stress resultants $N_{i j}$, transverse shear stress resultants $\left\{Q_{\theta \theta}, Q_{\varphi \varphi}\right\}$, and the stress couples $M_{i j}$, where $i, j \in\{\theta, \varphi\}$, by

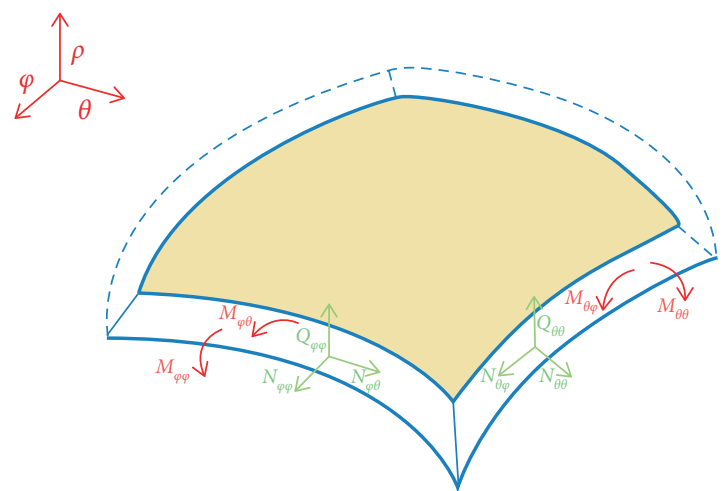

FIgURE 3: The stress resultants, transverse shear stress resultants, and stress couples on a differential element extracted from spherical shell.

integrating the infinitesimal stresses along the thickness of the spherical shell as [44].

Moreover, the stress resultants $\left(N_{\varphi \varphi}, N_{\theta \theta}\right.$, and,$\left.N_{\theta \varphi}\right)$ can be written as

$$
\begin{aligned}
& \left\{\begin{array}{c}
N_{\varphi \varphi} \\
N_{\theta \theta} \\
N_{\varphi \theta}=N_{\theta \varphi}
\end{array}\right\}=\int_{-h / 2}^{h / 2}\left\{\begin{array}{c}
\sigma_{\varphi \varphi} \\
\sigma_{\theta \theta} \\
\sigma_{\theta \varphi}
\end{array}\right\}\left(1+\frac{t}{R}\right) \mathrm{d} t, \\
& \left\{\begin{array}{c}
N_{\varphi \varphi} \\
N_{\theta \theta} \\
N_{\varphi \theta}=N_{\theta \varphi}
\end{array}\right\}=\frac{E h}{1-\nu^{2}}\left|\begin{array}{ccc}
1 & \nu & 0 \\
\nu & 1 & 0 \\
0 & 0 & \frac{(1-\nu)}{2}
\end{array}\right|\left\{\begin{array}{c}
\varepsilon_{\varphi \varphi}^{0} \\
\varepsilon_{\theta \theta}^{0} \\
2 \varepsilon_{\theta \varphi}^{0}
\end{array}\right\} .
\end{aligned}
$$

The stress couples can be expressed by

$$
\begin{gathered}
\left\{\begin{array}{c}
M_{\varphi \varphi} \\
M_{\theta \theta} \\
M_{\varphi \theta}=M_{\theta \varphi}
\end{array}\right\}=\int_{-h / 2}^{h / 2}\left\{\begin{array}{c}
\sigma_{\varphi \varphi} \\
\sigma_{\theta \theta} \\
\sigma_{\theta \varphi}
\end{array}\right\}\left(1+\frac{t}{R}\right) t \mathrm{~d} t, \\
\left.\left\{\begin{array}{c}
M_{\varphi \varphi} \\
M_{\theta \theta} \\
M_{\varphi \theta}=M_{\theta \varphi}
\end{array}\right\}=D\left|\begin{array}{ccc}
1 & v & 0 \\
\nu & 1 & 0 \\
0 & 0 & \frac{(1-\nu)}{2}
\end{array}\right| \mid \begin{array}{c}
\varepsilon_{\varphi \varphi}^{1} \\
\varepsilon_{\theta \theta}^{1} \\
2 \varepsilon_{\theta \varphi}^{1}
\end{array}\right\},
\end{gathered}
$$

where $D$ is the flexural rigidity defined as [17]

$$
D=\frac{E h^{3}}{12\left(1-v^{2}\right)}
$$

2.4. Stress Differential Equations of Motion. The stress differential equations of motion in the weightlessness can be expressed as $[39,40]$ 


$$
\begin{gathered}
\left(\sin \varphi N_{\varphi \varphi}\right)_{, \varphi}+\sin \varphi Q_{\varphi \varphi}-\cos \varphi N_{\theta \theta}+\left(N_{\varphi \theta}\right)_{, \theta}=m_{v} h R \sin \varphi \frac{\mathrm{d}^{2} u_{\varphi}}{\mathrm{d} t^{2}}, \\
\left(\sin \varphi N_{\theta \varphi}\right)_{, \varphi}+\left(N_{\theta \theta}\right)_{, \theta}+\cos \varphi N_{\varphi \theta}+\sin \varphi Q_{\theta \theta}=m_{v} h R \sin \varphi \frac{\mathrm{d}^{2} u_{\theta}}{\mathrm{d} t^{2}}, \\
\left(\sin \varphi Q_{\varphi \varphi}\right)_{, \varphi}-\sin \varphi N_{\varphi \varphi}-\sin \varphi N_{\theta \theta}+\left(Q_{\theta \theta}\right)_{, \theta}+R \sin \varphi p_{r}=m_{v} h R \sin \varphi \frac{\mathrm{d}^{2} w}{\mathrm{~d} t^{2}}, \\
\left(\sin \varphi M_{\varphi \varphi}\right)_{, \varphi}-R \sin \varphi Q_{\varphi \varphi}-\cos \varphi M_{\theta \theta}+\left(M_{\varphi \theta}\right)_{, \theta}=m_{v} \frac{h^{3}}{4} \sin \varphi \frac{d^{2} u_{\varphi}}{d t^{2}}-m_{v} \frac{h^{3}}{12} \sin \varphi \frac{\mathrm{d}^{2} w,_{\varphi}}{\mathrm{d} t^{2}} \\
\left(\sin \varphi M_{\theta \varphi}\right)_{, \varphi}+\left(M_{\theta \theta}\right)_{, \theta}+\cos \varphi M_{\varphi \theta}-R \sin \varphi Q_{\theta \theta}=m_{v} \frac{h^{3}}{4} \sin \varphi \frac{\mathrm{d}^{2} u_{\theta}}{\mathrm{d} t^{2}},
\end{gathered}
$$

where $m_{v}$ is the mass density of the material and $p_{r}$ is the received pressure perpendicular to the spherical shell.

\subsection{Derivation of Differential Equations for Axisymmetric} Vibrations. According to the torsionless axisymmetry with respect to the $z$-axis, we have

$$
\begin{array}{r}
u_{\theta}=\frac{\partial}{\partial \theta}(\cdot)=0 ; \\
N_{\varphi \theta}=N_{\theta \varphi}=N_{\rho \theta}=Q_{\theta \theta}=0 ; \\
M_{\varphi \theta}=M_{\theta \varphi}=0 .
\end{array}
$$

The compatibility relation can be deduced from (4), (5), and (12) $\left\{N_{\varphi \varphi}, N_{\theta \theta}\right\}$ by eliminating $u_{\theta}$ and replacing the strains by their expressions according to the stress resultants:

$$
\begin{aligned}
& \tan \varphi\left(N_{\theta \theta}-v N_{\varphi \varphi}\right)_{, \varphi}+\left(\sec ^{2} \varphi+\nu\right) N_{\theta \theta}-\left(\nu \sec ^{2} \varphi+1\right) N_{\varphi \varphi} \\
& =\frac{E h}{R} \tan \varphi\left[w_{, \varphi}+\tan \varphi w\right] .
\end{aligned}
$$

The expression of the shear stress resultant $Q_{\varphi \varphi}$ as a function of displacements $u_{\varphi}$ and $w$ can be obtained from (15) and (18):

$$
\begin{aligned}
Q_{\varphi \varphi}= & -\frac{D}{R}\left(\nabla^{2} w+\frac{2}{R^{2}} w\right)_{, \varphi}+m_{v} \frac{\left(1-v^{2}\right)}{R} \frac{D}{E} \frac{\mathrm{d}^{2} w,_{\varphi}}{\mathrm{d} t^{2}} \\
& -2 m_{v} \frac{\left(1-v^{2}\right)}{R} \frac{D}{E} \frac{\mathrm{d}^{2} u_{\varphi}}{\mathrm{d} t^{2}} .
\end{aligned}
$$

Inspired by [32], we introduce a new stress function $F(\varphi, t)$ through the following new relations:

$$
\begin{aligned}
N_{\varphi \varphi}= & \cot \varphi F_{, \varphi}+F-m_{v} \frac{(1+v)}{E} R^{2} \frac{\mathrm{d}^{2} F}{\mathrm{~d} t^{2}} \\
& +\frac{D}{R}\left(\nabla^{2} w+\frac{2}{R^{2}} w\right)-m_{v} \frac{\left(1-v^{2}\right)}{R} \frac{D}{E} \frac{\mathrm{d}^{2} w}{\mathrm{~d} t^{2}}
\end{aligned}
$$

$$
\begin{aligned}
N_{\theta \theta}= & F_{, \varphi \varphi}+F-m_{v} \frac{(1+v)}{E} R^{2} \frac{\mathrm{d}^{2} F}{\mathrm{~d} t^{2}} \\
& +\frac{D}{R}\left(\nabla^{2} w+\frac{2}{R^{2}} w\right)-m_{v} \frac{\left(1-v^{2}\right)}{R} \frac{D}{E} \frac{\mathrm{d}^{2} w}{\mathrm{~d} t^{2}}
\end{aligned}
$$

where, as proposed in [32], we have

$$
\nabla^{2} \equiv \frac{1}{R^{2}}\left[{ }_{, \varphi \varphi}+\cot \varphi_{, \varphi}\right]
$$

The tangential displacement $u_{\varphi}$ can be related to the radial displacement $w$, by elimination of $u_{\varphi}$ from (15) as

$$
u_{\varphi}=\tan \varphi\left[\frac{R}{E h}\left(\left(N_{\theta \theta}-\nu N_{\varphi \varphi}\right)\right)-w\right] .
$$

By using (4), (5), (15), (22), (23), and (24), we get the following differential equation:

$$
\frac{\mathrm{d}^{2} \Gamma}{\mathrm{d} t^{2}}=0
$$

where $\Gamma$ is expressed as

$$
\begin{aligned}
\Gamma= & R^{2} \nabla^{2} F+(1-v) F-m_{v}\left(1-v^{2}\right) \frac{R^{2}}{E} \frac{\mathrm{d}^{2} F}{\mathrm{~d} t^{2}} \\
& +(1-v) \frac{D}{R}\left(\nabla^{2}+\frac{2}{R^{2}}\right) w-\frac{E h}{R} w \\
& -m_{v}(1-v) \frac{\left(1-v^{2}\right)}{R} \frac{D}{E} \frac{\mathrm{d}^{2} w}{\mathrm{~d} t^{2}} .
\end{aligned}
$$

By substituting the equations (23) and (24) in the compatibility equation (21), another differential equation related also to $F$ and $w$ can be defined as

$$
\tan \varphi \Gamma+\Gamma_{, \varphi}=0 .
$$

The solution of (27) and (29) can be written as $\Gamma+A t+B=0$, where the coefficients $\mathrm{A}$ and $\mathrm{B}$ can be set to zero without loss in generality. Then, the first differential equation in $F$ and $w$ can be defined as 


$$
\begin{gathered}
R^{2} \nabla^{2} F+(1-v) F-m_{v}\left(1-v^{2}\right) \frac{R^{2}}{E} \frac{\mathrm{d}^{2} F}{\mathrm{~d} t^{2}} \\
+(1-v) \frac{D}{R}\left(\nabla^{2}+\frac{2}{R^{2}}\right) w-\frac{E h}{R} w \\
-m_{v}(1-v) \frac{\left(1-\nu^{2}\right)}{R} \frac{D}{E} \frac{\mathrm{d}^{2} w}{\mathrm{~d} t^{2}}=0 .
\end{gathered}
$$

To obtain the second differential equation in $F$ and $w$, the shear stress resultant $Q_{\varphi \varphi}$ (22) into (17) and using (23) and (24) can be substituted:

$$
\begin{gathered}
R^{2} \nabla^{2} F+2 F-2 m_{v}(1+v) \frac{R^{2}}{E}\left(1+\frac{h^{2}}{12} \nabla^{2}\right) \\
\cdot \frac{\mathrm{d}^{2} F}{\mathrm{~d} t^{2}} \mathrm{DR}\left(\nabla^{2}+\frac{2}{R^{2}}\right)\left(\nabla^{2}+\frac{2}{R^{2}}\right) w \\
+m_{v} R\left[h-\left(1-v^{2}\right) \frac{D}{E} \nabla^{2}\right] \frac{\mathrm{d}^{2} w}{\mathrm{~d} t^{2}}-R p_{r}=0 .
\end{gathered}
$$

\section{General Solution for Harmonic Axisymmetric Vibrations}

The steady-state problem of the spherical shells can be represented by the system of the two differential equations (30) and (31). Thus, the solution of this system in $F$ and $w$ can be written in the following form:

$$
\left\{\begin{array}{l}
F \\
w
\end{array}\right\}=\Re\left\{\begin{array}{l}
e^{j t \omega} F^{*} \\
e^{j t \omega} w^{*}
\end{array}\right\},
$$

where $w^{*}$ and $F^{*}$ are the magnitudes of the radial displacement $w$ and the stress function $F$ and $\omega$ is the circular frequency.

The natural frequency can be noted as

$$
\Omega^{2}=\frac{m_{v} \omega^{2} R^{2}}{E} .
$$

By eliminating $F^{*}$ from (30) and (31) and using the notation of the natural frequency, a differential equation in $w^{*}$ of the sixth-order can be obtained as

$$
\nabla^{6} w^{*}+k_{2} \nabla^{4} w^{*}+k_{3} \nabla^{2} w^{*}+k_{4} w^{*}+k_{5} R p_{r}=0
$$

where

$$
\begin{aligned}
& k_{2}=\frac{1}{R^{2}}\left[4+2 \Omega^{2}\left(1-v^{2}\right)\right], \\
& k_{3}=\frac{E h}{R^{2} D}\left(1-\Omega^{2}\right), \\
& k_{4}=\frac{E h}{\mathrm{DR}}\left[2+(1+3 v) \Omega^{2}-\left(1-v^{2}\right) \Omega^{4}\right], \\
& k_{5}=\frac{1}{D}\left[1+v+\Omega^{2}(1+v)^{2}\right] .
\end{aligned}
$$

In the particular case of the free vibration analysis, the surface loads are equal to zero. Equation (34) is then written as follows:

$$
\nabla^{6} w^{*}+k_{2} \nabla^{4} w^{*}+k_{3} \nabla^{2} w^{*}+k_{4} w^{*}=0 .
$$

Therefore, the general solution of the free vibration differential equation (36) can be expressed in terms of Legendre functions as

$$
w^{*}=\sum_{\alpha=1}^{3} w_{\alpha}^{*}
$$

where each radial displacement $w_{\alpha}^{*}$ can be written as

$$
w_{\alpha}^{*}=A_{\alpha} P_{n_{\alpha}}(\cos \varphi)+B_{\alpha} Q_{n_{\alpha}}(\cos \varphi), \quad \alpha \varepsilon\{1,2,3\},
$$

where $P_{n_{\alpha}}$ and $Q_{n_{\alpha}}$ are the Legendre functions of the first and the second kind of order $n_{\alpha}$, respectively. $n_{\alpha}$ can be defined as

$$
n_{\alpha}=\left(\lambda_{\alpha}+\frac{1}{4}\right)^{1 / 2}-\frac{1}{2} ; \quad \alpha \varepsilon\{1,2,3\} \text {. }
$$

With the aid of the identities,

$$
\nabla^{2}\left\{\begin{array}{c}
P_{n}(\cos \varphi) \\
Q_{n}(\cos \varphi)
\end{array}\right\}=-\frac{\lambda}{R^{2}}\left\{\begin{array}{c}
P_{n}(\cos \varphi) \\
Q_{n}(\cos \varphi)
\end{array}\right\} .
$$

The characteristic equation of the differential free vibrations equation of spherical shells (34) is

$$
\begin{aligned}
\lambda^{3}- & {\left[4+2 \Omega^{2}\left(1-v^{2}\right)\right] \lambda^{2}+12\left(1-\nu^{2}\right)\left(\frac{R}{h}\right)^{2}\left(1-\Omega^{2}\right) \lambda } \\
& -12\left(1-v^{2}\right)\left(\frac{R}{h}\right)^{2}\left[2+(1+3 \nu) \Omega^{2}+\left(\nu^{2}-1\right) \Omega^{4}\right]=0 .
\end{aligned}
$$

\section{Results and Discussion}

In this section, we test the validity and accuracy of the method exposed previously by determining the natural frequencies of spherical shells. Then, to demonstrate the correctness of the aforementioned formulation, two appropriate boundary conditions are treated: free edge and clamped edge. The current results are compared, on the one hand, with those available in literature, that is, the analytical results obtained by Naghdi-Kalnins [32] and by Kunieda [33]. It should be noted that these two analytical methods do not take into consideration the terms of the inertial couples in their equations of motion. On the other hand, the current results are also compared with the numerical results obtained by two different axisymmetric finite elements: the first one is SHELL61 that does not take into account transverse shear deformation and the second is SHELL209 that takes it into account. Furthermore, these two shell finite elements are also suitable for the modeling of axisymmetric thin shells. SHELL61 has two nodes and four degrees of freedom per node: displacements in the $x$ (radial), $y$ (axial), and $z$ (circular) directions and rotation around the $z$-axis. The loading may be axisymmetric or nonaxisymmetric $[45,46]$. 
SHELL209 has three nodes and three degrees of freedom per node: displacements in the $x, y$ directions and rotation about the $z$-axis [45]. More information on the shape functions is available in $[45,46]$. The FEM solutions are computed via ANSYS Mechanical APDL. The mesh is fine enough to guarantee the accuracy of the numerical results. In this study, only the thin shells whose thickness $h$ is less than the twentieth of the radius of curvature $R$ will be considered. The thickness to radius ratio in this work varies in the range 0.0025 to 0.05 .

\subsection{Free Vibration with Free Edge}

4.1.1. Frequency Equation. In this section, we focus on solving the problem of axisymmetric vibrations of a thin hemispherical shell with a free edge $\left(0 \leq \varphi \leq \varphi_{0}\right)$ with a free circular boundary at $\left(\varphi_{0}=\pi / 2\right)$.

In the case of thin hemispherical shells $(0 \leq \varphi \leq \pi / 2)$, the Legendre functions of the second kind $Q_{n_{\alpha}}$ can tend towards infinity for $(\varphi=0)$. To eliminate the singularity of $Q_{n_{\alpha}}$, the coefficients $B_{\alpha}$ are set to zero, where $\alpha \varepsilon\{1,2,3\}$ is as (38).

In the free edge boundary condition at $(\varphi=\pi / 2)$, the stress resultant $N_{\varphi \varphi}$, the stress couple $M_{\varphi \varphi}$, and the transverse shear stress resultant $Q_{\varphi \varphi}$ are set to zero $\left(N_{\varphi \varphi}=M_{\varphi \varphi}=Q_{\varphi \varphi}=0\right)$ :

$$
\sum_{\alpha=1}^{3} A_{\alpha}\left[\frac{E h}{(1-\nu) R}-\lambda_{\alpha} C_{\alpha} \frac{D}{R^{3}}\right] P_{n_{\alpha}}(0)=0
$$

$$
\begin{aligned}
& \sum_{\alpha=1}^{3} A_{\alpha} \frac{D}{R^{2}} \lambda_{\alpha}\left[1-\frac{1}{12}\left(\frac{h}{R}\right)^{2} C_{\alpha}\right] P_{n_{\alpha}}(0)=0, \\
& \sum_{\alpha=1}^{3} A_{\alpha} \frac{D}{R^{3}}\left[\lambda_{\alpha}-2-\left(1-v^{2}\right) \Omega^{2}+\frac{1}{6}\left(1-v^{2}\right) \Omega^{2} \frac{h^{2}}{R^{2}} C_{\alpha}\right] \\
& \cdot\left(n_{\alpha}+1\right) P_{n_{\alpha}+1}(0),
\end{aligned}
$$

where

$$
C_{\alpha}=\frac{\lambda_{\alpha}-2+\Omega^{2}\left(\nu^{2}-1\right)+12\left(R^{2} / h^{2}\right)(\nu+1)}{\left(\nu^{2}-1\right) \Omega^{2}+v+\lambda_{\alpha}-1},
$$

and the coefficients of the frequency equation

$$
\left|C_{i \alpha}\right|=0 ; \quad i, \alpha \in\{1,2,3\} .
$$

Then, they can be written as

$$
\begin{aligned}
C_{1 \alpha}= & {\left[12(1+\nu)-\frac{h^{2}}{R^{2}} C_{\alpha} \lambda_{\alpha}\right] P_{n_{\alpha}}(0), } \\
C_{2 \alpha}= & {\left[1+\nu-\lambda_{\alpha}\right] P_{n_{\alpha}}(0), } \\
C_{3 \alpha}= & {\left[\lambda_{\alpha}-2-\left(1-\nu^{2}\right) \Omega^{2}+\frac{1}{6}\left(1-v^{2}\right) \Omega^{2} \frac{h^{2}}{R^{2}} C_{\alpha}\right] } \\
& \cdot\left(n_{\alpha}+1\right) P_{n_{\alpha}+1}(0),
\end{aligned}
$$

where the coefficient $C_{2 \alpha}$ was obtained by the linear combination between the two equations (42) and (43).
The Legendre function of a complex degree can be calculated by the following integral [28]:

$$
P_{n}(0)=(n+2) \frac{2 \sqrt{2}}{\pi} \int_{0}^{\pi / 2} \cos \left(n+\frac{3}{2}\right) x \cos ^{1 / 2} x \mathrm{~d} x .
$$

To calculate the lowest natural frequency from equation (46), for $\nu=0.3$ and $\varphi_{0}=\pi / 2$, we specify the value of $h / R$ and $\Omega$ and then evaluate the determinant $\left|C_{i \alpha}\right|$ by incrementing $\Omega$. This procedure is repeated until the value of the determinant changes sign. Table 1 and Figure 4 present an example of this procedure, where $h / R=3 / 100$. As shown in Table 1, the real parts of the natural frequencies are negligible. For this reason, in this case, we consider just the imaginary part. Then, the small box of Figure 4 shows that there are two sign changes between 0.82 and $1.01 \mathrm{~Hz}$. Therefore, it means that in this interval, we have two natural frequencies.

It is worthy to mention that a determinant is a complex number, as shown in Table 2. In this case, the real parts of the determinants are not negligible. For this reason, we used the determinant's modulus to determine the lowest natural frequencies, which correspond to the minimum values closest to zero. The variation of the determinant's modulus as a function of natural frequency for $h / R=3 / 100$ and $\nu=$ 0.3 is also plotted in Figure 5. As seen in the zoomed panel, the results indicate the existence of five minimum values, which correspond to the lowest natural frequencies. Their values are presented in Table 3.

4.1.2. Comparison of Results. In order to verify the accuracy of the current solution, the results are compared with those obtained by Naghdi and Kalnins [32], Kunieda [33], and calculus by the finite elements SHELL61 and SHELL209. The difference estimators $\eta_{i}, i \in\{1,2,3,4\}$ are defined as follows:

$$
\begin{aligned}
& \eta_{1}=\frac{\left|\Omega_{\text {Kunieda [33] }}-\Omega_{\text {current }}\right|}{\Omega_{\text {Kunieda [33] }}} \times 100, \\
& \eta_{2}=\frac{\left|\Omega_{\text {Kunieda [33] }}-\Omega_{\text {Naghdi-Kalnins [32] }}\right|}{\Omega_{\text {Kunieda [33] }}} \times 100, \\
& \eta_{3}=\frac{\left|\Omega_{\text {Kunieda [33] }}-\Omega_{\mathrm{FE-SHELL61}}\right|}{\Omega_{\text {Kunieda [33] }}} \times 100, \\
& \eta_{4}=\frac{\left|\Omega_{\text {Kunieda [33] }}-\Omega_{\mathrm{FE}-\text { SHELL209 }}\right|}{\Omega_{\text {Kunieda [33] }}} \times 100,
\end{aligned}
$$

where $\Omega_{\text {Kunieda [33] }}$ is the frequency obtained by the Kunieda method, $\Omega_{\text {current }}$ is the frequency obtained by the current method, $\Omega_{\text {Naghdi-Kalnins [32] }}$ is the frequency obtained by the Naghdi-Kalnins method, $\Omega_{\mathrm{FE}-\mathrm{SHELL61}}$ is the frequency obtained by the FE-SHELL61, and $\Omega_{\mathrm{FE}-\mathrm{SHELL} 209}$ is the frequency obtained by the FE-SHELL209. It should be noted that the analytical results obtained by the Kunieda method $\Omega_{\text {Kunieda [33] }}$ are taken as a reference.

Table 4 shows a comparison study of the lowest natural frequency for spherical elastic thin shells under free 
TABLE 1: The current analytical value of determinant at $h / R=3 / 100$ and $v=0.3$ (free edge boundary condition).

\begin{tabular}{lccccc}
\hline$\Omega$ & \multicolumn{1}{c}{$\left|C_{i \alpha}\right|$} & $\Omega$ & $\left|C_{i \alpha}\right|$ & $\Omega$ & $\left|C_{i \alpha}\right|$ \\
\hline 0 & $3.6778 e-06-5.2305 e+10 i$ & 0.6097 & $2.8036 e-04-3.3459 e+12 i$ & 0.8738 & $7.3712 e-09+6.5295 e+07 i$ \\
0.0333 & $5.7014 e-12-3.8527 e+11 i$ & 0.6667 & $-1.3833 e-04-1.6127 e+12 i$ & 0.8745 & $1.8190 e-08+1.9857 e+08 i$ \\
0.0667 & $-2.2297 e-10-1.3537 e+12 i$ & 0.7224 & $-6.1685 e-05-6.2522 e+11 i$ & 0.878 & $-2.6839 e-08+7.8168 e+08 i$ \\
0.1667 & $-5.7854 e-04-6.8094 e+12 i$ & 0.7499 & $2.7660 e-05-3.4821 e+11 i$ & 0.9 & $-1.0285 e-07+2.1516 e+09 i$ \\
0.3333 & $2.0823 e-03-1.3573 e+13 i$ & 0.7860 & $8.8061 e-06-1.3605 e+11 i$ & 0.9568 & $-2.7725 e-08+2.8182 e+08 i$ \\
0.386 & $-6.4884 e-08-1.3043 e+13 i$ & 0.8 & $-7.0441 e-06-8.7855 e+10 i$ & 0.9839 & $1.3324 e-08-2.0230 e+08 i$ \\
0.416 & $7.4700 e-04-1.2178 e+13 i$ & 0.8221 & $5.1090 e-06-3.8812 e+10 i$ & 1.0 & $-7.4506 e-09-2.0294 e+08 i$ \\
0.5 & $-3.2127 e+04-7.1348 e+20 i$ & 0.8333 & $2.8874 e-08-2.3493 e+10 i$ & 1.0109 & $-7.4506 e-09-8.0483 e+07 i$ \\
\hline
\end{tabular}

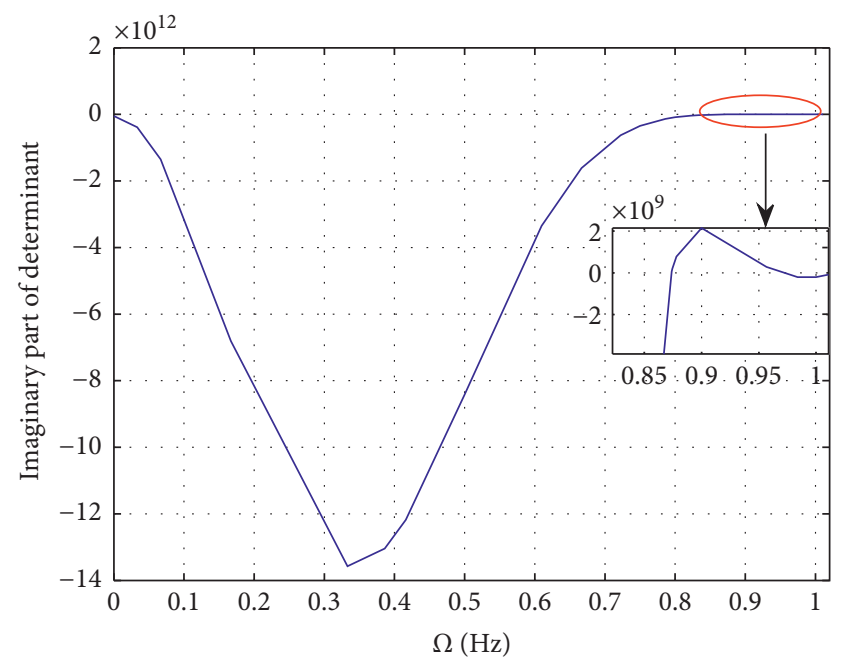

Figure 4: The imaginary part of determinant in variation with $\Omega$ at $h / R=3 / 100$ and $\nu=0.3$ (free edge boundary condition).

TABLE 2: Complex determinant in variation of $\Omega$ at $h / R=3 / 100$ and $\nu=0.3$ (free edge boundary condition).

\begin{tabular}{lccccc}
\hline$\Omega$ & \multicolumn{1}{c}{$\left|C_{i \alpha}\right|$} & $\Omega$ & $\left|C_{i \alpha}\right|$ & $\Omega$ & $\left|C_{i \alpha}\right|$ \\
\hline 0 & $3.6778 e-06-5.2305 e+10 i$ & 0.878 & $-2.6839 e-08+7.8168 e+08 i$ & 1.12 & $-4.7369 e+08+4.4042 e-07 i$ \\
0.0333 & $5.7014 e-12-3.8527 e+11 i$ & 0.9 & $-1.0285 e-07+2.1516 e+09 i$ & 1.122 & $-3.2406 e+08+2.3935 e-07 i$ \\
0.0667 & $-2.2297 e-10-1.3537 e+12 i$ & 0.9568 & $-2.7725 e-08+2.8182 e+08 i$ & 1.1245 & $-1.0195 e+08+1.7176 e-07 i$ \\
0.1667 & $-5.7854 e-04-6.8094 e+12 i$ & 0.9839 & $1.3324 e-08-2.0230 e+08 i$ & 1.1259 & $4.0270 e+07+2.7768 e-07 i$ \\
0.3333 & $2.1534 e-08-1.3574 e+13 i$ & 1.0 & $-7.4506 e-09-2.0294 e+08 i$ & 1.1270 & $1.6136 e+08+1.0339 e-07 i$ \\
0.386 & $-6.4884 e-08-1.3043 e+13 i$ & 1.0109 & $-7.4506 e-09-8.0483 e+07 i$ & 1.1287 & $3.6519 e+08+7.3535 e-10 i$ \\
0.416 & $7.4700 e-04-1.2178 e+13 i$ & 1.0222 & $-8.9407 e-08+1.3505 e+08 i$ & 1.14 & $2.2893 e+09+4.1215 e-07 i$ \\
0.5 & $-4.7887 e-04-8.4152 e+12 i$ & 1.0603 & $-2.9802 e-08+9.4023 e+08 i$ & 1.16 & $8.6272 e+09+5.5198 e-07 i$ \\
0.6097 & $2.8036 e-04-3.3459 e+12 i$ & 1.0820 & $-1.1921 e-07+6.4678 e+08 i$ & 1.17 & $1.3452 e+10+1.5723 e-06 i$ \\
0.6667 & $-1.3833 e-04-1.6127 e+12 i$ & 1.0830 & $2.9802 e-08+5.9779 e+08 i$ & 1.2 & $3.5002 e+10+4.7824 e-06 i$ \\
0.7224 & $-6.1685 e-05-6.2522 e+11 i$ & 1.085 & $-2.0862 e-07+4.7795 e+08 i$ & 1.23 & $6.4097 e+10-8.6780 e-06 i$ \\
0.7499 & $2.7660 e-05-3.4821 e+11 i$ & 1.089 & $-2.1815 e+08-1.1882 e-08 i$ & 1.25 & $8.2221 e+10+5.2564 e-06 i$ \\
0.7860 & $8.8061 e-06-1.3605 e+11 i$ & 1.100 & $-8.4835 e+08+6.4085 e-08 i$ & 1.27 & $9.2099 e+10+1.0384 e-05 i$ \\
0.8 & $-7.0441 e-06-8.7855 e+10 i$ & 1.1100 & $-8.9064 e+08+1.2904 e-07 i$ & 1.29 & $8.4653 e+10+8.1133 e-06 i$ \\
0.8221 & $5.1090 e-06-3.8812 e+10 i$ & 1.1126 & $-8.3109 e+08+1.6805 e-07 i$ & 1.31 & $4.8412 e+10-6.7699 e-06 i$ \\
0.8333 & $2.8874 e-08-2.3493 e+10 i$ & 1.1162 & $-6.9389 e+08+7.3412 e-08 i$ & 1.4 & $-8.2261 e+11+1.3543 e-04 i$ \\
0.8738 & $7.3712 e-09+6.5295 e+07 i$ & 1.1187 & $-5.5821 e+08+9.8344 e-08 i$ & 1.5 & $-3.2889 e+12-4.0268 e-04 i$ \\
\hline
\end{tabular}

vibration with free edge boundary condition for $h / R=1 / 100$ and $v=0.3$, calculated by the current method, the Naghdi-Kalnins method [32], and the Kunieda method [33]. We observe that the current results and Kunieda's ones are identical. It is also seen that the Naghdi-Kalnins results are very close to these results, with $\eta_{2}=0.57 \%$.
A comparative study of the first seventeen natural frequencies between the analytical solutions (the current solution and Kunieda's [33] solution) and numerical solutions (FE-SHELL61 and FE-SHELL209), is carried out in Table 5 for thin spherical shells under free vibration with the free edge boundary condition at $h / R=1 / 100$ and $\nu=0.3$. 


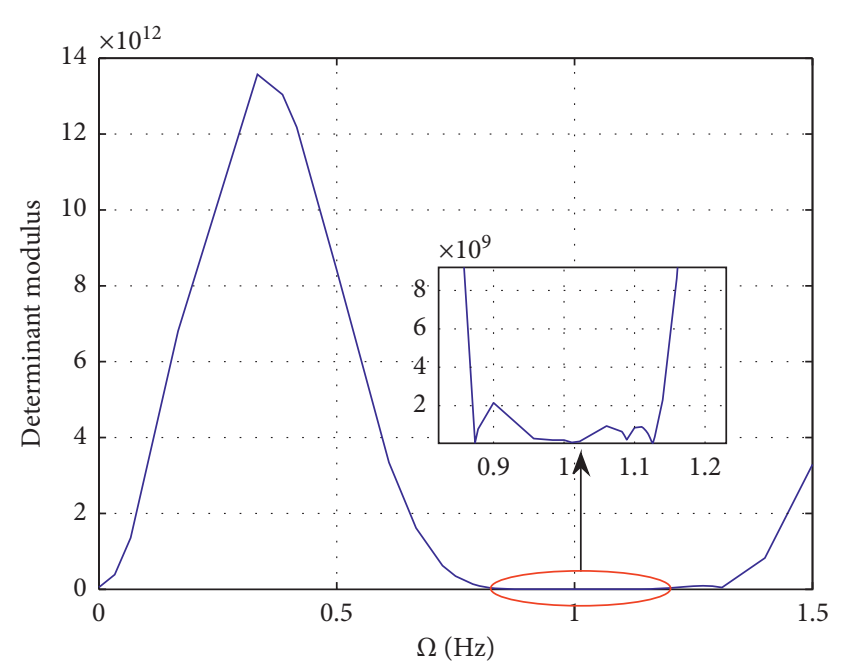

FIgURE 5: Determinant modulus in variation with $\Omega$ at $h / R=3 / 100$ and $\nu=0.3$ (free edge boundary condition).

TABle 3: The lowest natural frequencies for axisymmetric free vibrations, under free edge boundary condition, with the current analytical methods at $h / R=3 / 100$ and $\nu=0.3$.

\begin{tabular}{lc}
\hline Mode number & $\Omega_{\text {Current }}$ \\
\hline 1 & 0.873 \\
2 & 0.983 \\
3 & 1.011 \\
4 & 1.089 \\
5 & 1.126 \\
6 & 1.310 \\
\hline
\end{tabular}

TABLE 4: The lowest natural frequency for axisymmetric free vibrations, under free edge boundary condition, with different methods at $h / R=1 / 100$ and $\nu=0.3$.

\begin{tabular}{lcccc}
\hline$\Omega_{\text {Current }}$ & $\Omega_{\text {Naghdi-Kalnins [32] }}$ & $\Omega_{\text {Kunieda [33] }}$ & $\eta_{1}(\%)$ & $\eta_{2}(\%)$ \\
\hline 0.870 & 0.875 & 0.870 & 0.00 & 0.57 \\
\hline
\end{tabular}

From Table 5, we observe a very good agreement between the different results confirming the accuracy of the current analytical method. On the one hand, we see that the current results are close to the results of Kunieda's method. The difference between the results of these two analytical methods is very small and does not exceed $0.27 \%$. This closeness is verified even for higher vibration modes. On the other hand, we see also that the FE-SHELL61 results are very close to the SHELL209 results particularly for the first modes. Indeed, when the ratio $h / R$ is very small (here $h / R=1 / 100$ ) the transverse shear becomes negligible and the two finite elements give almost the same results. We observe also that, in order, the current results are closer, first, to the results of the finite element SHELL61, then to those of Kunieda, and finally to those of the finite element SHELL209.

The results of Table 5 are instructive in more than one way. The current method reveals a new natural frequency 1.042 ( $6^{\text {th }}$ mode) which does not appear in Kunieda's method and finite element calculation while the frequency $1.961\left(13^{\text {th }}\right.$ mode) obtained by the finite element SHELL61 is nonexistent for the two analytical calculations and the finite element SHELL209 calculation.

4.2. Effect of the Thickness to Radius Ratio $h / R$ on the Lowest Natural Frequency. The results in Table 6, which are plotted in Figure 6, show the effect of the $h / R$ ratio on the lowest natural frequency of thin spherical shells under free vibrations with the free edge. We observe that the lowest frequency, calculated by the current method and FE methods, is almost independent of the $h / R$ ratio, while that calculated by the Naghdi-Kalnins method is sensitive to this ratio. This strong variation in frequency, calculated by the Naghdi-Kalnins method, as a function of the $h / R$ ratio is not legitimate.

All the methods give almost the same lowest frequency when the ratio $h / R$ is very small.

We also observe that the two finite element methods give exactly the same lowest frequency even for high values of the ratio $h / R$. Which leads us to say that taking into account the transverse shear does not have effect on the lowest frequency and therefore that the current method can be applied in this case even for high $h / R$ ratios.

\subsection{Free Vibrations with Clamped Edge Boundary Condition}

4.3.1. Frequency Equation. For free vibrations with clamped boundary conditions, the edge $\left(\varphi=\varphi_{0}\right)$ of the shell cannot translate in the $\rho$ and $\varphi$ directions and cannot undergo any rotation around the $e_{\theta}$ axis. The boundary conditions, in this case, can be summarized as

$$
\begin{gathered}
\left.w\right|_{\varphi=\varphi_{0}} ^{*}=0, \\
\frac{d w}{\mathrm{~d} t} l_{\varphi=\varphi_{0}}^{*}=0, \\
u_{\varphi}^{*} /_{\varphi=\varphi_{0}}=0,
\end{gathered}
$$

where $\left(\varphi_{0}=\pi / 2\right)$. Also, in this case of clamped boundary conditions, the Legendre functions of the second kind $Q_{n_{\alpha}}$ can tend towards infinity for $(\varphi=0)$. To eliminate the singularity of $Q_{n_{\alpha}}$, the coefficients $B_{\alpha}$ are set to zero as mentioned in the free vibrations with free edge condition section.

Using the boundary conditions (50) to (52) along with the expression for $u_{\varphi}^{*}$, the following form is obtained:

$$
\begin{aligned}
\sum_{\alpha=1}^{3} A_{\alpha} P_{n_{\alpha}}(0) & =0 \\
\sum_{\alpha=1}^{3} A_{\alpha}\left(n_{\alpha}+1\right) P_{n_{\alpha}+1}(0) & =0 \\
\sum_{\alpha=1}^{3} A_{\alpha} C_{\alpha}\left(n_{\alpha}+1\right) P_{n_{\alpha}+1}(0) & =0 .
\end{aligned}
$$

We note that the displacement $u_{\varphi}^{*}$ can be expressed as a function of $F_{, \varphi}$ (as derived in the appendix). 
TABLE 5: Comparison study for natural frequencies for axisymmetric free vibrations, under free edge boundary condition, with different methods at $h / R=1 / 100$ and $\nu=0.3$.

\begin{tabular}{|c|c|c|c|c|c|c|c|c|c|c|c|c|c|c|c|c|c|}
\hline $\begin{array}{l}\text { Mode number } \\
\text { method }\end{array}$ & $1^{\text {st }}$ & $2^{\text {nd }}$ & $3^{\text {rd }}$ & $4^{\text {th }}$ & $5^{\text {th }}$ & $6^{\text {th }}$ & $7^{\text {th }}$ & $8^{\text {th }}$ & $9^{\text {th }}$ & $10^{\text {th }}$ & $11^{\text {th }}$ & $12^{\text {th }}$ & $13^{\text {th }}$ & $14^{\text {th }}$ & $15^{\text {th }}$ & $16^{\text {th }}$ & $17^{\text {th }}$ \\
\hline Current & 0.870 & 0.952 & 0.983 & 1.001 & 1.029 & 1.042 & 1.078 & 1.151 & 1.254 & 1.389 & 1.559 & 1.761 & & 1.996 & 2.070 & 2.262 & 2.559 \\
\hline Kunieda [33] & 0.870 & 0.952 & 0.983 & 1.001 & 1.030 & & 1.079 & 1.152 & 1.255 & 1.391 & 1.561 & 1.765 & & 2.001 & 2.069 & 2.268 & \\
\hline FE-SHELL61 & 0.870 & 0.952 & 0.982 & 1.000 & 1.029 & & 1.077 & 1.151 & 1.254 & 1.389 & 1.558 & 1.761 & 1.961 & 1.995 & 2.070 & 2.261 & 2.557 \\
\hline FE-SHELL209 & 0.870 & 0.952 & 0.982 & 1.000 & 1.029 & & 1.077 & 1.150 & 1.252 & 1.386 & 1.553 & 1.753 & & 1.984 & 2.070 & 2.245 & 2.534 \\
\hline$\eta_{1}(\%)$ & 0.000 & 0.000 & 0.000 & 0.000 & 0.097 & & 0.092 & 0.086 & 0.079 & 0.143 & 0.128 & 0.226 & & 0.249 & 0.048 & 0.264 & \\
\hline$\eta_{3}(\%)$ & 0.000 & 0.000 & 0.101 & 0.099 & 0.097 & & 0.185 & 0.086 & 0.079 & 0.143 & 0.192 & 0.226 & & 0.299 & 0.048 & 0.308 & \\
\hline$\eta_{4}(\%)$ & 0.000 & 0.000 & 0.101 & 0.099 & 0.097 & & 0.185 & 0.173 & 0.239 & 0.359 & 0.512 & 0.679 & & 0.849 & 0.048 & 1.014 & \\
\hline
\end{tabular}

TABLE 6: Variation of the lowest natural frequency for axisymmetric free vibrations, under free edge boundary condition, as a function of ratio $h / R$ with different methods at $\nu=0.3$.

\begin{tabular}{lcccc}
\hline$h / R$ ratio & $\Omega_{\text {Naghdi-Kalnins[32] }}$ & $\Omega_{\text {Current }}$ & $\Omega_{\text {FE-SHELL61 }}$ & $\Omega_{\text {FE-SHELL209 }}$ \\
\hline 0.01 & 0.875 & 0.870 & 0.870 & 0.870 \\
0.02 & 0.919 & 0.871 & 0.871 & 0.871 \\
0.03 & 0.975 & 0.873 & 0.874 & 0.874 \\
0.05 & 1.100 & 0.879 & 0.879 & 0.879 \\
\hline
\end{tabular}

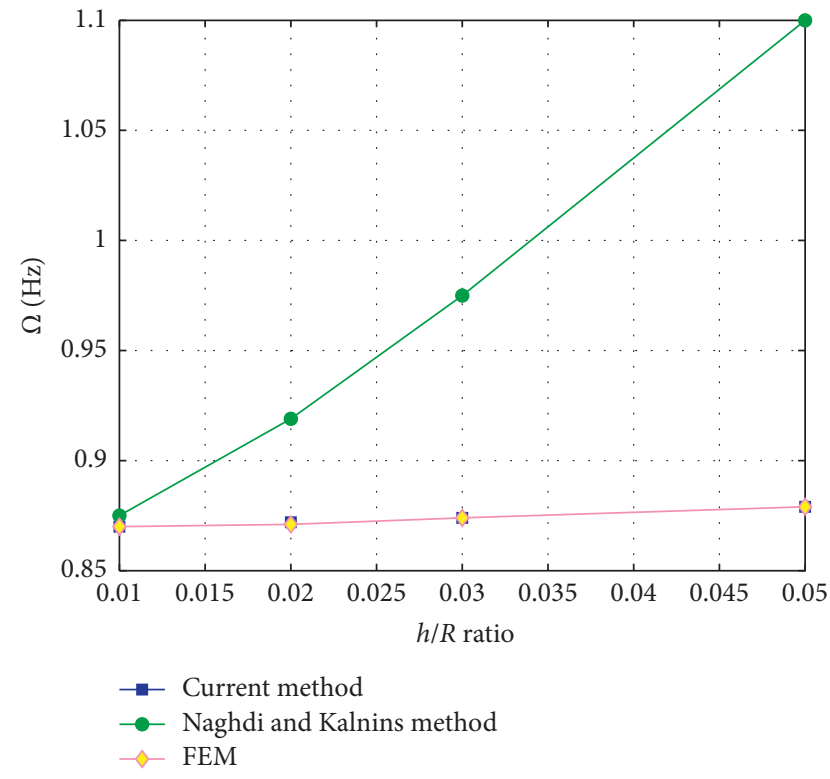

FIGURE 6: Natural frequency as a function of thickness to radius ratio $h / R$ at $\nu=0.3$ (free edge boundary condition).

The coefficients of the frequency equation:

$$
\left|C_{i \alpha}\right|=0 ;, \quad i, \alpha \in\{1,2,3\},
$$

and they can be written as

$$
\begin{aligned}
& C_{1 \alpha}=P_{n_{\alpha}}(0), \\
& C_{2 \alpha}=\left(n_{\alpha}+1\right) P_{n_{\alpha}+1}(0), \\
& C_{3 \alpha}=C_{\alpha}\left(n_{\alpha}+1\right) P_{n_{\alpha}+1}(0) .
\end{aligned}
$$

To calculate lowest natural frequency from equation (54) for $\nu=0.3$ and $\varphi_{0}=\pi / 2$, we specify the value of $h / R$ and $\Omega$
TABLE 7: The lowest natural frequencies for axisymmetric free vibrations, under clamped edge boundary condition, with current analytical method at $h / R=3 / 100$ and $\nu=0.3$.

\begin{tabular}{lc}
\hline Mode number & $\Omega_{\text {Current }}$ \\
\hline 1 & 0.782 \\
2 & 0.973 \\
3 & 1.088 \\
4 & 1.099 \\
\hline
\end{tabular}

and then evaluate the determinant $\left|C_{i \alpha}\right|$ by incrementing $\Omega$ as done in the section of frequency equation for free vibrations with free edge.

Table 7 presents the lowest natural frequencies for thin spherical shells at $h / R=3 / 100$ and $v=0.3$. The results are obtained by the current analytical method under axisymmetric free vibrations with clamped edge boundary condition.

4.3.2. Comparison of Results. In order to verify the accuracy of the current method, in the case of axisymmetric free vibrations for hemispherical thin shells with clamped edge boundary condition at $h / R=1 / 100$ and $v=0.3$, the frequencies of the first sixteen modes are compared with those obtained by Kunieda [33] and calculus by the finite elements SHELL61 and SHELL209, as shown in Table 8.

The current solutions are in close agreement with the solutions obtained by Kunieda, FE-SHELL61, and FESHELL209. The difference between the results obtained by the current method and Kunieda and between the current method and FE-SHELL61 are closely zero. For the higher modes, a negligible difference appears in the results of FESHELL209 compared to the others results, probably due to the fact that it takes into account the transverse shear.

The results of Table 8 are instructive in more than one way. The current method reveals a new natural frequency $1.042\left(5^{\text {th }}\right.$ mode) which does not appear in Kunieda's method and in the the finite element calculation while the 
TABLE 8: Comparison of natural frequencies for axisymmetric free vibrations, under clamped edge boundary condition, with different methods at $h / R=1 / 100$ and $\nu=0.3$.

\begin{tabular}{|c|c|c|c|c|c|c|c|c|c|c|c|c|c|c|c|c|}
\hline Mode number & $1^{\text {st }}$ & $2^{\text {nd }}$ & $3^{\mathrm{rd}}$ & $4^{\text {th }}$ & $5^{\text {th }}$ & $6^{\text {th }}$ & $7^{\text {th }}$ & $8^{\text {th }}$ & $9^{\text {th }}$ & $10^{\text {th }}$ & $11^{\text {th }}$ & $12^{\text {th }}$ & $13^{\text {th }}$ & $14^{\text {th }}$ & $15^{\text {th }}$ & $16^{\text {th }}$ \\
\hline Current & 0.761 & 0.938 & 0.983 & 1.021 & 1.042 & 1.070 & 1.144 & & 1.246 & 1.379 & 1.541 & 1.660 & 1.775 & 1.998 & 2.261 & 2.552 \\
\hline Kunieda [33] & 0.760 & 0.938 & 0.984 & 1.020 & & 1.071 & 1.144 & & 1.247 & 1.381 & 1.542 & 1.660 & 1.777 & 2.002 & 2.266 & \\
\hline FE-SHELL61 & 0.761 & 0.937 & 0.983 & 1.020 & & 1.070 & 1.143 & 1.240 & 1.245 & 1.379 & 1.539 & 1.660 & 1.774 & 1.998 & 2.260 & 2.552 \\
\hline FE-SHELL209 & 0.761 & 0.937 & 0.983 & 1.019 & & 1.069 & 1.142 & & 1.243 & 1.375 & 1.534 & 1.657 & 1.767 & 1.985 & 2.242 & 2.527 \\
\hline$\eta_{1}(\%)$ & 0.131 & 0 & 0.101 & 0.098 & & 0.093 & 0 & & 0.080 & 0.144 & 0.064 & 0 & 0.112 & 0.199 & 0.220 & \\
\hline$\eta_{3}(\%)$ & 0.131 & 0.106 & 0.101 & 0 & & 0.093 & 0.087 & & 0.561 & 0.144 & 0.194 & 0 & 0.168 & 0.199 & 0.264 & \\
\hline$\eta_{4}(\%)$ & 0.131 & 0.106 & 0.101 & 0.098 & & 0.186 & 0.174 & & 0.320 & 0.434 & 0.518 & 0.180 & 0.562 & 0.849 & 1.059 & \\
\hline
\end{tabular}

TABLE 9: Influence of ratio $h / R$ on the lowest natural frequencies for axisymmetric free vibrations, under clamped edge boundary condition, with different methods at $\nu=0.3$.

\begin{tabular}{|c|c|c|c|c|c|}
\hline$h / R$ ratio & Mode number & $\Omega_{\text {Current }}$ & $\Omega_{\text {Kunieda [33] }}$ & $\Omega_{\text {SHELL61 }}$ & $\Omega_{\text {SHELL209 }}$ \\
\hline \multirow{4}{*}{0.0025} & 1 & 0.747 & 0.747 & 0.746 & 0.747 \\
\hline & 2 & 0.929 & 0.929 & 0.929 & 0.929 \\
\hline & 3 & 0.968 & 0.968 & 0.968 & 0.968 \\
\hline & 4 & 0.983 & 0.983 & 0.983 & 0.983 \\
\hline \multirow{4}{*}{0.005} & 1 & 0.753 & 0.752 & 0.753 & 0.753 \\
\hline & 2 & 0.932 & 0.932 & 0.932 & 0.932 \\
\hline & 3 & 0.972 & 0.972 & 0.971 & 0.971 \\
\hline & 4 & 0.991 & 0.991 & 0.990 & 0.990 \\
\hline \multirow{4}{*}{0.01} & 1 & 0.761 & 0.760 & 0.761 & 0.761 \\
\hline & 2 & 0.938 & 0.938 & 0.937 & 0.937 \\
\hline & 3 & 0.983 & 0.984 & 0.938 & 0.983 \\
\hline & 4 & 1.020 & 1.020 & 1.020 & 1.020 \\
\hline \multirow{5}{*}{0.02} & 1 & 0.773 & 0.772 & 0.773 & 0.773 \\
\hline & 2 & 0.953 & 0.953 & 0.952 & 0.952 \\
\hline & 3 & 1.028 & 1.029 & 1.027 & 1.027 \\
\hline & 4 & 1.067 & & & \\
\hline & 5 & 1.130 & & 1.130 & 1.129 \\
\hline
\end{tabular}

frequency 1.240 obtained by the FE-SHELL61 is nonexistent for the three other methods.

4.3.3. Influence of the Thickness to Radius Ratio $h / R$. The results in Table 9 show the effect of the $h / R$ ratio on the four (or five) lowest natural frequencies of thin spherical shells under free vibrations with clamped edge.

We observe that, for ratio $h / R$, varying between 0.0025 and 0.02 , the natural frequencies obtained by the current method are very close to those obtained by Kunieda's method and those of the calculation by finite elements.

The effect of transverse shear is nonexistent even for the highest ratio $h / R=0.02$ : the element SHELL209 which takes account of the transverse shear gives the same results. Therefore, the current method can be applied in this case even for high $h / R$ ratios and even for high modes, with negligible error as observed above.

The effect of the $h / R$ ratio is weak on the lowest frequency. The variation of this frequency is 3.5\% when the $h / R$ ratio varies from the value 0.0025 to 0.02 while the influence of the $h / R$ ratio on the frequency of mode 4 is more important. The variation of this frequency is $8.5 \%$, when the $h / R$ ratio varies in the same interval. Kunieda [33] considers that the effect of $h / R$ is not substantial for thin shells.
This effect is much greater for thin shells with a clamped edge than for those with a free edge. When the $h / R$ ratio varies from the value 0.01 to 0.02 , the percentage variation of the lowest frequency is $1.6 \%$ for clamped edge and only $0.1 \%$ for free edge.

The current method reveals a new natural frequency (1.067, mode 4) which is not obtained by the finite element calculus and which is absent from Kunieda's results.

\section{Conclusions}

The present method solves the differential equations of the axisymmetric vibrations of elastic thin spherical shells. These differential equations of motion in terms of stress take into account the terms of inertial couples in the equations of stress couples. These terms are omitted in the analytical methods proposed by Naghdi-Kalnins and by Kunieda. A new stress function is introduced and new equations as a function of the radial displacement and the stress function are derived. The harmonic axisymmetric free vibrations are described by a sixth-order differential equation in terms of the radial displacement $w$, the natural frequency $\Omega$, the thickness to radius ratio of the shell $h / R$, and Poisson's ratio $\nu$. The general solution is expressed by the Legendre function of degree $n_{\alpha}$. Taking into account the boundary conditions 
makes it possible to reach the analytical solution in the specific case of the axisymmetric harmonic vibrations of the hemispherical shell. In this work, we tested two boundary conditions: free and clamped edges.

The results demonstrate the correctness and high accuracy of the current analytical method compared with the Naghdi-Kalnins and Kunieda analytical methods and the finite element calculation.

This method is distinguished by taking into account the inertia of rotation (inertial couple in the stress couples' differential equation of motion) in addition to the two inertias of translation while the methods of Naghdi-Kalnins and Kunieda only take into account the two inertias of translation, which probably explains the evidence by this method of new frequencies. This method can be applied, with good approximation, for the calculation of the first natural frequencies even for moderately high values of $h / R$ ratios for free edge or clamped edge cases, despite the fact that it does not take into account the effect of the transverse shear.

It can also be extremely useful for the validation of numerical methods.

\section{Appendix}

\section{A: Proof of the Tangential Displacement $u_{\varphi}$}

Substituting the stress resultant expressions (23) and (24) in the expression (26) of the tangential displacement $u_{\varphi}$, then, by using the first differential equation in terms of $F$ and $w$ as in (30), the tangential displacement can be written as

$$
u_{\varphi}=-(1+v) \frac{R}{E h} F_{, \varphi} .
$$

Using equations (30), (31), and (37), the stress function $F^{*}$ can be expressed in terms of the radial displacement $w^{*}$ as $F^{*}=\frac{E h}{R} \sum_{\alpha=1}^{3}\left[\frac{\left(\left[\lambda_{\alpha}-2-\Omega^{2}\left(1-\nu^{2}\right)\right](h / R)^{2} / 12(1+\nu)\right)+1}{\left[-\lambda_{\alpha}+(1-\nu)+\Omega^{2}\left(1-\nu^{2}\right)\right]}\right] w_{\alpha}^{*}$.

From (A.2) and (38), it can be then found that

$$
\begin{aligned}
F_{, \varphi}^{*}= & \frac{E h}{R} \sum_{\alpha=1}^{3}\left[\frac{\left(\left[\lambda_{\alpha}-2-\Omega^{2}\left(1-\nu^{2}\right)\right](h / R)^{2} / 12(1+\nu)\right)+1}{\left[-\lambda_{\alpha}+(1-\nu)+\Omega^{2}\left(1-\nu^{2}\right)\right]}\right] \\
& \times A_{\alpha}\left(n_{\alpha}+1\right)\left[\frac{1}{\sin \varphi} P_{n_{\alpha}+1}(\cos \varphi)-\frac{\cos \varphi}{\sin \varphi} P_{n_{\alpha}}(\cos \varphi)\right] .
\end{aligned}
$$

After substituting (A.3) in (A.1), the tangential displacement can be expressed as

$$
\begin{aligned}
u_{\varphi}^{*}= & -(1+\nu) \sum_{\alpha=1}^{3}\left[\frac{\left(\left[\lambda_{\alpha}-2-\Omega^{2}\left(1-v^{2}\right)\right](h / R)^{2} / 12(1+\nu)\right)+1}{\left[-\lambda_{\alpha}+(1-\nu)+\Omega^{2}\left(1-\nu^{2}\right)\right]}\right] \\
& \times A_{\alpha}\left(n_{\alpha}+1\right)\left[\frac{1}{\sin \varphi} P_{n_{\alpha}+1}(\cos \varphi)-\frac{\cos \varphi}{\sin \varphi} P_{n_{\alpha}}(\cos \varphi)\right] .
\end{aligned}
$$

\section{Data Availability}

No data were used to support this study.

\section{Conflicts of Interest}

The authors declare no conflicts of interest.

\section{Acknowledgments}

The authors would like to thank Mr. Vishal Gupta for his technical support.

\section{References}

[1] M. Glück, M. Breuer, F. Durst, A. Halfmann, and E. Rank, "Computation of fluid-structure interaction on lightweight structures," Journal of Wind Engineering and Industrial Aerodynamics, vol. 89, no. 14-15, pp. 1351-1368, 2001.

[2] V. Crupi, G. Epasto, and E. Guglielmino, "Comparison of aluminium sandwiches for lightweight ship structures: honeycomb vs. foam," Marine Structures, vol. 30, pp. 74-96, 2013.

[3] J. Rex and B. Kameshwari, "Studies on pumice lightweight aggregate concrete with quarry dust using mathematical modeling aid of ACO techniques," Advances in Materials Science and Engineering, vol. 2016, 2016.

[4] M. L. Wallach, "Smart sensor systems-submarine marking and sonar detection," US Patent 7,839,305, 2010.

[5] M. Caresta and N. J. Kessissoglou, "Acoustic signature of a submarine hull under harmonic excitation," Applied Acoustics, vol. 71, no. 1, pp. 17-31, 2010.

[6] A. L. Kalamkarov and I. I. Andrianov, "Analytical solution of the stability problem for the truncated hemispherical shell under tensile loading," Mathematical Problems in Engineering, vol. 2018, Article ID 5260639, , 2018.

[7] M. Nassit and H. Berbia, "Structures simulation of curved sensor based on PVDF for fetal heart rate monitoring," in Proceedings of the 2018 IEEE/ACS 15th International Conference on Computer Systems and Applications (AICCSA), pp. 1-6, Aqaba, Jordan, November 2018.

[8] H. Li, F. Pang, and H. Chen, "A semi-analytical approach to analyze vibration characteristics of uniform and stepped annular-spherical shells with general boundary conditions," European Journal of Mechanics-A/Solids, vol. 74, pp. 48-65, 2019.

[9] H. Razavi, A. F. Babadi, and Y. Tadi Beni, "Free vibration analysis of functionally graded piezoelectric cylindrical nanoshell based on consistent couple stress theory," Composite Structures, vol. 160, pp. 1299-1309, 2017.

[10] Y. Kiani, "Free vibration of FG-CNT reinforced composite spherical shell panels using Gram-Schmidt shape functions," Composite Structures, vol. 159, pp. 368-381, 2017.

[11] F. Léonard, J. Lanteigne, S. Lalonde, and Y. Turcotte, "Freevibration behaviour of a cracked cantilever beam and crack detection," Mechanical Systems and Signal Processing, vol. 15, no. 3, pp. 529-548, 2001

[12] B. Akgöz and Ö. Civalek, "Free vibration analysis of axially functionally graded tapered Bernoulli-Euler microbeams based on the modified couple stress theory," Composite Structures, vol. 98, pp. 314-322, 2013.

[13] Z. Yang, S. Guo, J. Yang, and Y. Hu, "On the eigenvalue problem for free vibrations of a piezoelectric/piezomagnetic body," IEEE Transactions on Ultrasonics, Ferroelectrics, and Frequency Control, vol. 55, pp. 734-737, 2008. 
[14] G. Giunta, Y. Koutsawa, S. Belouettar, and H. Hu, "Static, free vibration and stability analysis of three-dimensional nanobeams by atomistic refined models accounting for surface free energy effect," International Journal of Solids and Structures, vol. 50, no. 9, pp. 1460-1472, 2013.

[15] F. Pang, H. Li, J. Cui, Y. Du, and C. Gao, "Application of flügge thin shell theory to the solution of free vibration behaviors for spherical-cylindrical-spherical shell: a unified formulation," European Journal of Mechanics-A/Solids, vol. 74, pp. 381-393, 2019.

[16] Y. Kiani and M. R. Eslami, "The GDQ approach to thermally nonlinear generalized thermoelasticity of a hollow sphere," International Journal of Mechanical Sciences, vol. 118, pp. 195-204, 2016.

[17] F. Sammoura, S. Akhbari, and L. Lin, "An analytical solution for curved piezoelectric micromachined ultrasonic transducers with spherically shaped diaphragms," IEEE Transactions on Ultrasonics, Ferroelectrics, and Frequency Control, vol. 61, no. 9, pp. 1533-1544, 2014.

[18] M. Javani, Y. Kiani, M. Sadighi, and M. R. Eslami, "Nonlinear vibration behavior of rapidly heated temperature-dependent FGM shallow spherical shells," AIAA Journal, vol. 57, no. 9, pp. 4071-4084, 2019.

[19] X. Chen and K. Ye, "Free vibration analysis for shells of revolution using an exact dynamic stiffness method," Mathematical Problems in Engineering, vol. 2016, 2016.

[20] Y. Kiani, M. Sadighi, and M. R. Eslami, "Dynamic analysis and active control of smart doubly curved FGM panels," Composite Structures, vol. 102, pp. 205-216, 2013.

[21] A. E. H. Love, "The small free vibrations and deformation of a thin elastic shell," Philosophical Transactions of the Royal Society of London A, pp. 491-546, 1888.

[22] J. L. Batoz and G. Dhatt, Poutres et Plaques, Hermès, Paris, France, 1993.

[23] R. Naz and F. Mahomed, "Dynamic euler-Bernoulli beam equation: classification and reductions," Mathematical Problems in Engineering, vol. 2015, Article ID 520491, , 2015.

[24] E. Reissner, Thin-Shell Structures: Theory, Experiment, and Design, Prentice-Hall, Upper Saddle River, NJ, USA, 1974.

[25] R. Mindlin, "Influence of rotatory inertia and shear on flexural motions of isotropic, elastic plates," Journal of Applied Mechanics, vol. 18, pp. 31-38, 1951.

[26] E. Reissner, "The effect of transverse shear deformation on the bending of elastic plates," Journal of Applied Mechanics, pp. A69-A77, 1945.

[27] E. Reissner, Reflections on the Theory of Elastic Plates, 1985.

[28] P. Naghdi, "Foundations of elastic shell theory," in Progress in Solid Mechanics, I. N. Sneddon, Ed., vol. 4North-Holland, Netherlands, 1963.

[29] H. Kraus, Thin Elastic Shells, 1967.

[30] S. P. Timoshenko, Résistance des matériaux, 1968.

[31] J. N. Reddy and C. F. Liu, "A higher-order shear deformation theory of laminated elastic shells," International Journal of Engineering Science, vol. 23, no. 3, pp. 319-330, 1985.

[32] P. M. Naghdi and A. Kalnins, "On vibrations of elastic spherical shells," Journal of Applied Mechanics, vol. 29, no. 1, pp. 65-72, 1962.

[33] H. Kunieda, "Solutions of free vibrations of spherical shells: Part 3 natural frequencies and modes in axi and anti-symmetric state," Transactions of the Architectural Institute of Japan, vol. 325, pp. 57-66, 1983.

[34] H. Kunieda, "Flexural axisymmetric free vibrations of a spherical dome: exact results and approximate solutions," Journal of Sound and Vibration, vol. 92, no. 1, pp. 1-10, 1984.
[35] N. Perrone and R. Kao, "A general finite difference method for arbitrary meshes," Computers \& Structures, vol. 5, no. 1, pp. 45-57, 1975.

[36] B. A. Finlayson, The Method of Weighted Residuals and Variational Principles, SIAM, Delhi, India, 2013.

[37] S. H. Gould, Variational Methods for Eigenvalue Problems: An Introduction to the Methods of Rayleigh, Ritz, Weinstein, and Aronszajn, Courier Corporation, Chelmsford, MA, USA, 2012.

[38] O. C. Zienkiewicz and P. Morice, The Finite Element Method in Engineering Science, McGraw-Hill, London, UK, 1971.

[39] A. El Harif, Étude par la méthode des éléments finis de l'effet de cisaillement transverse dans les plaques et les coques de revolution; Mémoire de thèse de doctorat d'état. Ph.D. thesis, Faculty of Science, Mohammed V University, Rabat, Morocco, 1988.

[40] J. L. Batoz and G. Dhatt, Modélisation des Structures par Éléments Finis Volume 3 Coques, HERMES, Paris, France, 1992.

[41] Q. H. Jebur, "Dynamic analysis of thin composite cylindrical and spherical shells," Al-Khwarizmi Engineering Journal, vol. 4, pp. 69-75, 2008.

[42] Y. Du, F. Pang, L. Sun, and H. Li, “A unified formulation for dynamic behavior analysis of spherical cap with uniform and stepped thickness distribution under different edge constraints," Thin-Walled Structures, vol. 146, p. 106445, 2020.

[43] A. Bryan, "Free vibration of doubly curved thin shells," Journal of Vibration and Acoustics, vol. 140, 2018.

[44] J. N. Reddy, Theory and Analysis of Elastic Plates and Shells, CRC Press, Boca Raton, FL, USA, 2006.

[45] I. Ansys, ANSYS Mechanical APDL Element Reference, ANSYS, Inc., Canonsburg, PA, USA, 2011.

[46] I. Ansys, "Theory reference for the mechanical APDL and mechanical applications," Southpointe Release, vol. 12, 2009. 